\section{Mental disorders and employment status in the São Paulo Metropolitan Area, Brazil: gender differences and use of health services}

Transtornos mentais e situação laboral na Região Metropolitana de São Paulo, Brasil: diferenças de gênero e uso de serviços de saúde

\author{
Trastornos mentales y situación laboral en \\ la zona metropolitana de São Paulo, Brasil: \\ diferencias de género y uso de \\ servicios de salud
}

\author{
Mariane Henriques França 1 \\ Sandhi Maria Barreto 2 \\ Flavia Garcia Pereira 1 \\ Laura Helena Silveira Guerra de Andrade 3 \\ Maria Cristina Alochio de Paiva 1 \\ Maria Carmen Viana 1
}

doi: 10.1590/0102-311X00154116

\begin{abstract}
Mental disorders are associated with employment status as significant predictors and as consequences of unemployment and early retirement. This study describes the estimates and associations of 12-month DSM-IV prevalence rates of mental disorders and use of health services with employment status by gender in the São Paulo Metropolitan Area, Brazil. Data from the São Paulo Megacity Mental Health Survey was analyzed $(n=5,037)$. This is a population-based study assessing the prevalence and determinants of mental disorders among adults, using the Composite International Diagnostic Interview. The associations were estimated by odds ratios obtained through binomial and multinomial logistic regression. This study demonstrates that having mental disorders, especially mood disorders, is associated with being inactive or unemployed among men and inactive among women, but only having a substance use disorder is associated with being unemployed among women. Among those with mental disorders, seeking health care services is less frequent within unemployed.
\end{abstract}

Mental Disorders; Employment; Health Services

\author{
Correspondence \\ M. H. França \\ Programa de Pós-graduação em Saúde Coletiva, Universidade \\ Federal do Espírito Santo. \\ Av. Marechal Campos 1468, Vitoria, ES 29092-030, Brasil. \\ marianehf@yahoo.com.br \\ 1 Programa de Pós-graduação em Saúde Coletiva, Universidade \\ Federal do Espírito Santo, Vitória, Brasil. \\ 2 Faculdade de Medicina, Universidade Federal de Minas \\ Gerais, Belo Horizonte, Brasil. \\ 3 Faculdade de Medicina, Universidade de São Paulo, \\ São Paulo, Brasil.
}




\section{Background}

Neuropsychiatric disorders are the leading cause of disability in the world 1,2. Results from the Global Burden of Disease (GBD) in Brazil showed that neuropsychiatric conditions were responsible for 21.5\% of all disability-adjusted life years (DALYs) (25.1\% among women and 18.6\% among men), generating high social and economic costs 3,4. Around the world, several studies were launched to estimate the prevalence and determinants of mental disorders in the general population, as well as to assess the impact of mental disorders on daily-living activities and work performance 5,6,7,8,9,10,11,12,13,14,15,16,17, including in Brazil 18. Most mental disorders have early onset, often leading to precocious and/or permanent disability, increasing the need for health care 15,16,17,18,19,20,21. In Brazil, mental disorders are the third leading cause of disability leaves from work and disability benefits 22 .

Mental disorders are significantly associated with unemployment and early retirement due to permanent or temporary disability 6,7,9,10,15,16,17,18,19,20. Low treatment-seeking rates reduce adequate rehabilitation and subsequent return to the workforce 7,9,10,14,15,16,22,23. These associations, though, are likely to be two-way, as unemployment and its adverse consequences may deleteriously impact mental health $6,7,9,12,15,16,18,19$.

High prevalence rates of psychiatric morbidity were reported among adult residents living in the São Paulo Metropolitan Area, Brazil 24,25, where mood, substance use and anxiety disorders, altogether, accounted for $49 \%$ of the total days out of role in the previous month 18 .

The present study further explores the associations of 12-month common mental disorders with employment status in the adult population in the São Paulo Metropolitan Area, and its relation to gender and use of health care services, using data from the São Paulo Megacity Mental Health Survey 26.

\section{Methods}

The São Paulo Megacity Mental Health Survey is a population-based cross-sectional study of psychiatric morbidity in the general adult population (18+) living in the São Paulo Metropolitan Area, comprising the capital of the state of São Paulo and 38 surrounding municipalities, and covering a geographical area of $8,501 \mathrm{~km}^{2} .5,037$ subjects were assessed in their households, with a global response rate of 81.3\%. Data were collected between May/2005 and April/2007 by trained lay interviewers, using the World Mental Health (WMH) Survey version of the Composite International Diagnostic Interview (CIDI 3.0) 27, which is composed of clinical and non-clinical sections. Detailed information on the study design and methods are available elsewhere26.

\section{Measurements and outcomes}

\section{- Sociodemographic characteristics}

The following characteristics were assessed: (1) age at interview, grouped into 4 categories (18-34, $35-49,50-64$, or 65+ years); (2) gender, female or male; (3) education, grouping the number of years of schooling into 4 categories (0-4, 5-8, 8-11; and 12+ years); (4) marital status, as currently married/ cohabitating, separated/widowed/divorced, and never married; (5) monthly income within quintiles, in Brazilian currency (low income mean = BRL 1,217; low-middle mean = BRL 3,056; middle mean = BRL 5,055; high-middle mean = BRL 8,438; and high income mean = BRL 26,481); according to the Brazilian Ministry of Labor 28, the minimum monthly income at the time of data collection was BRL 300,00 (from may/2005 to april/2006), and BRL 350,00 (from May/2006 to April/2007).

\section{- Mental disorders}

To estimate the 12-month prevalence of 19 DSM-IV mental disorders (12m-MD) according to employment status by gender, nineteen mental disorders occurring in the 12 months prior to the interview (12m-MD) were assessed through the CIDI 3.0 clinical sections based on the Diagnostic and Statistical Manual of Mental Disorders, 4th Edition (DSM-IV) diagnostic criteria 26. Mental disorders 
considered here include mood disorders (major depression, I/II bipolar disorders, and dysthymia); anxiety disorders (agoraphobia, generalized anxiety disorder, obsessive compulsive disorder, panic disorder, post-traumatic stress disorder, adult separation anxiety disorder, social phobia, and specific phobia); impulse-control disorders (attention deficit disorder, conduct disorder, oppositional defiant disorder, and intermittent explosive disorder); substance use disorders (SUD) (alcohol abuse, alcohol dependence, drug abuse, and drug dependence).

\section{- Current employment status}

To describe the distribution of employment status and its association with $12 \mathrm{~m}-\mathrm{MD}$ by gender, respondents were grouped into 3 categories according to their employment situation at the time of the interview: (1) working (currently working, self-employed, and/or in maternity leave; $\mathrm{n}=3,007$ ); (2) economically inactive (retirees, homeworkers, students, illness/sickness leave, and/or disabled; $\mathrm{n}=1,487$ ); and (3) unemployed (looking for work, currently unemployed, and/or temporarily laid off; $\mathrm{n}=521)$. Twenty-six respondents answered to an open-ended question and were reclassified accordingly, e.g., "construction worker without a contract" or "provider of laundry/ironing services" were considered as Working. 107 respondents chose more than one option and were classified as "working" if they earned any money for the work they provided, e.g., one respondent was a student and had a part-time unregistered occupation; or a homeworker who also worked as baby-sitter. Information regarding employment status is missing for 22 respondents, which were excluded from analyses (total $\mathrm{N}=5,015)$.

\section{- $\quad$ Treatment seeking}

In an attempt to verify if employment status is related to use of health care services among respondents with $12 \mathrm{~m}-\mathrm{MD}$, treatment-seeking information was analyzed. Seeking specialized treatment ("any mental health treatment") was considered if respondents saw any mental healthcare professionals in the past 12 months, including psychiatrists and psychologists, or were admitted into a psychiatric hospital/ward. "Any general health treatment" included attendance to any family doctor, other specialty physicians or other medical professionals in the past 12 months.

\section{Statistical analysis}

Descriptive statistics and measurements of associations were calculated applying survey sampling procedures that consider the complex sampling design, using weights to adjust for sampling probabilities and for age-gender structure of the target-population, using Stata (StataCorp LP, College Station, USA). Descriptive analysis of sociodemographic characteristics was conducted according to employment status. The association between $12 \mathrm{~m}-\mathrm{MD}$ and employment status was estimated by the Pearson's chi-squared test and the magnitude of the associations by odds ratio (OR) and its $95 \%$ confidence interval (95\%CI), obtained through multinomial logistic regression, with "working" as the reference category, stratified by gender, and further adjusted by age, education and marital status. We also used binomial logistic regression to assess whether treatment seeking was associated with employment status among those with any $12 \mathrm{~m}-\mathrm{MD}$, adjusted by age, education, marital status and comorbidity of mental disorders. A significant level of 0.05 was considered in all tests.

\section{Results}

At the time of the interview, $63.6 \%(\mathrm{n}=3,007)$ of the sample were currently working, $24.6 \%(\mathrm{n}=$ $1,487)$ economically inactive, and $11.8 \%(n=521)$ unemployed (Table 1$)$. There were significant differences in gender distributions across current employment status groups; women were 5.5 times more likely to be economically inactive (95\%CI: 4.38-6.90; p < 0.01), and had 35\% more chance of being unemployed (95\%CI: 1.09-1.66; $\mathrm{p}<0.01$ ), when compared to men (results not shown in the table, but available upon request). Most working and unemployed respondents were young (18-34 years); 
Sociodemographic characteristics of respondents by employment status in the São Paulo Megacity Mental Health Survey, Brazil $(N=5,015)$.

\begin{tabular}{|c|c|c|c|}
\hline & \multicolumn{3}{|c|}{ Employment status [\% (SE)] } \\
\hline & $\begin{array}{l}\text { Currently working } \\
(\mathrm{n}=3,007 ; 63.6 \%)\end{array}$ & $\begin{array}{c}\text { Inactive } \\
(n=1,487 ; 24.6 \%)\end{array}$ & $\begin{array}{c}\text { Unemployed } \\
(n=521 ; 11.8 \%)\end{array}$ \\
\hline \multicolumn{4}{|l|}{ Gender } \\
\hline Female & $44.1(1.15)$ & $75.1(1.36)$ * & $53.1(2.63)$ * \\
\hline Male ** & $55.9(1.15)$ & $24.9(1.36)$ & $46.9(2.63)$ \\
\hline \multicolumn{4}{|l|}{ Age groups (years) } \\
\hline $18-34 * *$ & $48.4(1.09)$ & $25.2(1.33)$ & $66.8(2.65)$ \\
\hline $35-49$ & $36.5(0.92)$ & $23.3(1.36)$ & $24.7(2.57)$ * \\
\hline $50-64$ & $12.8(0.53)$ & $25.3(1.51)$ * & $8.5(1.58)$ \\
\hline$\geq 65$ & $2.3(3.80)$ & $26.1(1.68)$ * & $0.0(0.00)$ \\
\hline \multicolumn{4}{|l|}{ Years of education } \\
\hline $0-4$ ** & $15.9(0.68)$ & $39.1(0.16)$ & $17.9(2.87)$ \\
\hline $5-8$ & $23.0(0.11)$ & $24.9(0.1)$ & $24.3(2.30)$ \\
\hline $9-11$ & $39.3(0.11)$ & $26.4(2.26)$ & $50.0(3.24)$ \\
\hline 12 & $21.8(0.96)$ & $9.6(0.92)$ * & $7.8(1.32) * \star \star$ \\
\hline \multicolumn{4}{|l|}{ Marital status } \\
\hline Married/Cohabitating ** & $60.0(1.32)$ & $66.6(1.60)$ & $39.6(2.36)$ \\
\hline Separated/Widowed/Divorced & $13.0(0.86)$ & $21.3(0.98)$ * & $15.0(1.33) * \star *$ \\
\hline Never mkarried & $27.0(1.18)$ & $12.1(1.37)$ * & $45.4(2.72)$ * \\
\hline \multicolumn{4}{|l|}{ Monthly income per capita (BRL) } \\
\hline High (1st quintile) ** & $23.6(0.01)$ & $18.1(1.10)$ & $6.9(1.56)$ \\
\hline High-middle (2nd quintile) & $22.9(0.90)$ & $16.1(1.10)$ * & $9.2(1.42)$ \\
\hline Middle (3rd quintile) & $19.6(7.60)$ & $17.5(0.93)$ * & $16.7(2.36) * * \star$ \\
\hline Low-middle (4th quintile) & $18.7(0.70)$ & $25.1(0.01) * * *$ & $31.3(3.10)$ * \\
\hline Low (5th quintile) & $15.1(0.56)$ & $23.2(1.31)$ * & $35.8(3.19)$ * \\
\hline
\end{tabular}

SE: standard error.

* p-value < 0.05;

** Within each employment status group;

*** $\mathrm{p}$-value $<0.001$ (Pearson's chi-squared tests, considering the reference category.

older cohorts were more likely to be economically inactive. Respondents with higher education were more likely to be working than economically inactive or unemployed. There were more separated and never married respondents among the economically inactive and the unemployed, and more married respondents among those currently working. Those with lower income were more likely to be economically inactive and especially unemployed, as a direct result of the lack of earnings (Table 1).

There were no differences in the prevalence of any DSM-IV $12 \mathrm{~m}-\mathrm{MD}$ across current employment status groups among men (Table 2) or among women (Table 3). When adjusting for sociodemographic characteristics, having any $12 \mathrm{~m}-\mathrm{MD}(\mathrm{OR}=1.5 ; 95 \% \mathrm{CI}$ : $1.01-2.29)$, any anxiety disorders $(\mathrm{OR}=1.8$; 95\%CI: 1.04-3.06), and specifically panic (OR $=4.4$; 95\%CI: $1.07-17.7)$, and post-traumatic stress disorder $(\mathrm{OR}=7.7 ; 95 \% \mathrm{CI}$ : 1.20-50.06) were associated with being economically inactive among men (Table 2). Moreover, the presence of any 12-month mood disorders increased the chances of being both economically inactive and unemployed $(\mathrm{OR}=2.8$; 95\%CI: 1.22-6.54 and OR = 1.7; 95\%CI: 1.01 2.82, respectively), as observed for bipolar disorder $(\mathrm{OR}=17.8 ; 95 \% \mathrm{CI}$ : 4.37-72.74 and $\mathrm{OR}=4.7$; 95\%CI: 1.26-17.78); major depressive disorder was significantly associated with being unemployed $(\mathrm{OR}=2.0$; 95\%CI: 1.20-3.24).

Among women, having bipolar disorder ( $\mathrm{OR}=3.6$; 95\%CI: $1.19-11.47)$, social phobia $(\mathrm{OR}=2.2$; 95\%CI: 1.29-3.63), and oppositional defiant disorder (OR =6.9; 95\%CI: 1.47-32.25) were associated 


\section{Table 2}

Prevalence of 12-month DSM-IV mental disorders and association with employment status among men in the São Paulo Megacity Mental Health Survey, Brazil $(\mathrm{N}=2,185)$

\begin{tabular}{|c|c|c|c|c|}
\hline \multirow[t]{3}{*}{ Disorder class/DSM-IV disorder } & \multicolumn{4}{|c|}{ Employment status } \\
\hline & \multirow{2}{*}{$\begin{array}{c}\text { Working * }(n=1,606) \\
\text { Weighted } \\
{[\%(S E)]}\end{array}$} & \multicolumn{3}{|c|}{ Inactive ** $(n=368)$} \\
\hline & & $\begin{array}{l}\text { Weighted } \\
\text { [\% (SE)] }\end{array}$ & Crude OR $(95 \% \mathrm{Cl}) * * *$ & $\begin{array}{l}\text { Adjusted OR } \\
\text { (95\%Cl) \# }\end{array}$ \\
\hline Any mental disorder & $20.6(1.30)$ & $20.7(2.55)$ & $1.0(0.76-1.33)$ & 1.5 (1.01-2.29) \#\# \\
\hline Comorbidity (> 1 mental disorders) & $5.9(0.64)$ & $9.1(2.54)$ & $1.6(0.81-3.09)$ & 2.5 (1.14-5.68) \#\# \\
\hline \multicolumn{5}{|l|}{ Mood disorders } \\
\hline Any mood disorder & $5.2(0.65)$ & $9.01(2.54)$ & $1.8(0.95-3.41)$ & $2.8(1.22-6.54) \# \#$ \\
\hline Major depression & $4.8(0.62)$ & $6.6(1.91)$ & $1.4(0.73-2.78)$ & $1.9(0.78-4.73)$ \\
\hline I/II bipolar disorder & $0.4(0.17)$ & $2.4(1.37)$ & $5.6(1.27-24.48) \# \#$ & 17.8 (4.37-72.74) \#\#\# \\
\hline Dysthymia & $0.5(0.24)$ & $2.0(1.55)$ & $3.8(0.49-29.71)$ & $5.7(0.82-39.30)$ \\
\hline \multicolumn{5}{|l|}{ Anxiety disorders } \\
\hline Any anxiety disorder & $12.5(1.02)$ & $13.3(3.00)$ & $1.3(0.69-2.36)$ & $1.2(0.68-2.02) \# \#$ \\
\hline Agoraphobia & $0.6(0.32)$ & $2.2(1.25)$ & $3.2(1.00-10.07)$ & $5.5(0.90-33.00)$ \\
\hline Generalized anxiety disorder & $1.5(0.37)$ & $1.7(0.67)$ & $1.1(0.44-2.88)$ & $1.8(0.67-4.80)$ \\
\hline Obsessive compulsive disorder & $3.0(0.52)$ & $2.7(1.06)$ & $0.9(0.34-2.15)$ & $1.2(0.39-3.38)$ \\
\hline Panic disorder & $0.5(0.14)$ & $0.6(0.37)$ & $1.2(0.30-4.87)$ & 4.4 (1.07-17.69) \#\# \\
\hline Post-traumatic stress disorder & $0.5(0.29)$ & $2.4(1.30)$ & $5.1(1.00-25.84)$ & 7.7 (1.20-50.06) \#\# \\
\hline Adult separation anxiety disorder & $1.6(0.40)$ & $2.9(2.02)$ & $1.8(0.40-9.10)$ & $1.6(0.23-11.61)$ \\
\hline Social phobia & $2.5(0.45)$ & $4.2(1.86)$ & $1.7(0.53-5.46)$ & $2.8(0.79-9.95)$ \\
\hline Specific phobia & $5.6(0.71)$ & $8.3(1.60)$ & $1.5(0.94-2.47)$ & $1.5(0.76-3.06)$ \\
\hline \multicolumn{5}{|l|}{ Impulse-control disordes } \\
\hline Any impulse-control disorder & $4.7(0.62)$ & $3.8(1.45)$ & $0.8(0.35-1.82)$ & $1.7(0.73-3.79)$ \\
\hline Attention deficit disorder & $1.2(0.34)$ & $1.8(1.20)$ & $1.6(0.38-6.47)$ & $2.9(0.56-14.47)$ \\
\hline Conduct disorder & $0.7(0.21)$ & $1.3(1.15)$ & $1.9(0.27-13.06)$ & $4.5(0.96-20.83)$ \\
\hline Oppositional defiant disorder & $0.4(0.20)$ & $0.5(0.40)$ & $1.5(0.18-11.64)$ & $1.3(0.28-5.81)$ \\
\hline Intermittent explosive disorder & $3.3(0.61)$ & $1.8(1.10)$ & $0.5(0.13-2.22)$ & $1.2(0.23-5.89)$ \\
\hline \multicolumn{5}{|l|}{ Substance use disorders } \\
\hline Any substance use disorder & $5.7(0.77)$ & $4.2(1.26)$ & $0.7(0.33-1.57)$ & $1.2(0.48-3.25)$ \\
\hline Alcohol abuse & $3.1(0.48)$ & $0.9(0.60)$ & $0.3(0.07-1.16)$ & $0.6(0.15-2.56)$ \\
\hline Alcohol dependence & $1.9(0.48)$ & $3.3(1.15)$ & $1.7(0.70-4.28)$ & $2.4(0.64-9.14)$ \\
\hline Drug abuse & $0.5(0.21)$ & $0.0(0.00)$ & - & - \\
\hline Drug dependence & $0.8(0.30)$ & $0.2(0.19)$ & $0.2(0.24-2.03)$ & $0.4(0.04-4.08)$ \\
\hline
\end{tabular}

(continues)

with being inactive. Any substance use disorder was associated with being unemployed $(\mathrm{OR}=2.3$; 95\%CI: 1.02-5.09), but this association lost statistical significance when adjusted for sociodemographic characteristics.

Regarding comorbidity, having more than one psychiatric disorders in the past 12 months was associated with being economically inactive (OR $=2.5$; 95\%CI: $1.14-5.68)$ and unemployed $(\mathrm{OR}=$ 1.5; 95\%CI: 1.0-2.38) among men, but was not related to current employment status among women.

A total of 1,395 respondents were diagnosed with at least one DSM-IV 12m-MD. Overall treatment-seeking rates in all health care settings are low, regardless of employment status. After adjusting for sociodemographic characteristics and for psychiatric comorbidities, those economically inactive were more likely to seek general health treatment (OR 3.0; CI 1.95-3.86), as well as mental health treatment $(\mathrm{OR}=2.8 ; 95 \% \mathrm{CI}: 1.51-5.12)$, when compared to those working (Table 4). Unemployed respondents with $12 \mathrm{~m}-\mathrm{MD}$ reported similar rates of general and specialized health care use compared to those working. 
Table 2 (continued)

\begin{tabular}{|c|c|c|c|}
\hline \multirow[t]{2}{*}{ Disorder class/DSM-IV disorder } & \multicolumn{3}{|c|}{$\begin{array}{l}\text { Employment status } \\
\text { Unemployed }(n=211)\end{array}$} \\
\hline & $\begin{array}{l}\text { Weighted } \\
\text { [\% (SE)] }\end{array}$ & $\begin{array}{l}\text { Weighted } \\
{[\%(95 \% \mathrm{Cl})]}\end{array}$ & $\begin{array}{l}\text { Weighted } \\
{[\%(95 \% \mathrm{Cl})]}\end{array}$ \\
\hline Any mental disorder & $25.8(2.82)$ & $1.3(0.94-1.89)$ & $1.2(0.82-1.84)$ \\
\hline Comorbidity (> 1 mental disorders) & $8.7(1.41)$ & $1.5(1.00-2.40)$ & $1.5(1.00-2.38)$ \\
\hline \multicolumn{4}{|l|}{ Mood disorders } \\
\hline Any mood disorder & $8.5(1.43)$ & $1.7(1.08-2.59) \# \#$ & $1.7(1.01-2.82) \# \#$ \\
\hline Major depression & $8.9(1.29)$ & $1.9(1.12-3.24) \# \#$ & $2.0(1.20-3.24) \# \#$ \\
\hline I/II bipolar disorder & $1.6(1.05)$ & $5.1(0.94-27.82)$ & $4.7(1.26-17.78) \# \#$ \\
\hline Dysthymia & $0.4(0.38)$ & $0.9(0.15-6.10)$ & $0.8(0.14-4.24)$ \\
\hline \multicolumn{4}{|l|}{ Anxiety disorders } \\
\hline Any anxiety disorder & $13.3(3.0)$ & $1.3(0.68-2.36)$ & $1.2(0.68-2.02)$ \\
\hline Agoraphobia & $0.3(0.24)$ & $0.4(0.06-2.98)$ & $0.2(0.02-1.70)$ \\
\hline Generalized anxiety disorder & $1.7(1.38)$ & $1.1(0.17-6.73)$ & $1.1(0.21-5.92)$ \\
\hline Obsessive compulsive disorder & $2.5(1.46)$ & $0.9(0.27-3.00)$ & $0.9(0.32-3.03)$ \\
\hline Panic disorder & $0.2(0.15)$ & $0.3(0.31-3.11)$ & $0.6(0.04-8.64)$ \\
\hline Post-traumatic stress disorder & $0.8(0.70)$ & $1.9(0.19-19.50)$ & $1.1(0.11-11.27)$ \\
\hline Adult separation anxiety disorder & $2.9(2.02)$ & $1.8(0.4-9.10)$ & $1.6(0.23-11.61)$ \\
\hline Social phobia & $3.6(1.45)$ & $1.4(0.57-3.61)$ & $1.7(0.68-4.33)$ \\
\hline Specific phobia & $6.2(2.03)$ & $1.1(0.54-2.29)$ & $0.9(0.49-2.02)$ \\
\hline \multicolumn{4}{|l|}{ Impulse-control disordes } \\
\hline Any impulse-control disorder & $4.2(1.55)$ & $0.9(0.36-2.15)$ & $0.9(0.31-2.46)$ \\
\hline Attention deficit disorder & $0.2(0.16)$ & $0.1(0.01-1.30)$ & $0.1(0.01-1.35)$ \\
\hline Conduct disorder & $0.7(0.61)$ & $1.1(0.17-6.86)$ & $0.6(0.05-8.72)$ \\
\hline Oppositional defiant disorder & $0.5(0.40)$ & $1.5(0.18-11.64)$ & $1.3(0.28-5.81)$ \\
\hline Intermittent explosive disorder & $3.1(0.48)$ & $0.9(0.31-2.81)$ & $0.9(0.27-3.25)$ \\
\hline \multicolumn{4}{|l|}{ Substance use disorders } \\
\hline Any substance use disorder & $8.1(2.34)$ & $1.3(0.60-2.87)$ & $1.0(0.41-2.57)$ \\
\hline Alcohol abuse & $4.4(1.96)$ & $1.4(0.52-3.88)$ & $1.3(0.40-4.15)$ \\
\hline Alcohol dependence & $3.7(1.68)$ & $1.9(0.56-6.73)$ & $1.2(0.24-5.54)$ \\
\hline Drug abuse & $0.4(0.15)$ & $0.6(0.13-2.55)$ & $0.4(0.39-4.73)$ \\
\hline Drug dependence & $0.5(0.31)$ & $0.7(0.65-8.59)$ & $0.3(0.08-1.31)$ \\
\hline
\end{tabular}

95\%Cl: 95\% confidence interval; DSM-IV: Diagnostic and Statistical Manual of Mental Disorders, 4th edition; OR: odds ratio; SE: standard error.

* Employed, self-employed, owner; OR = 1 (base-line);

** Homeworkers, students, disabled, retirees, sickness benefits;

*** OR cruded comparing inactive vs. working;

\# OR adjusted by sociodemographics characteristics comparing Inactive vs Working; OR obtained by multinomial logistic regression using the category "working" as the reference;

\#\# p-value < 0.05;

$\# \#$ p-value $<0.01$

\section{Discussion}

This study provides empirical information on the 12-month prevalence and associations of 19 DSMIV mental disorders with current employment status, according to gender, in the adult general population living in São Paulo, the largest metropolitan area in Brazil and in in all of South America. It further assessed the association between treatment seeking and employment status among respondents with 12-month DSM-IV mental disorders. Although these associations have been largely reported worldwide 5,6,7,8,9,10,11,12,13,14,15,16,17,19.18,20,21,23, there is little information of this sort in Brazil 18,22.

Regarding sociodemographic characteristics according to the current employment situation, our results are similar to reports from other populations $6,7,8,9,10,11,12$. Generally, working is associ- 


\section{Table 3}

Prevalence of 12-month DSM-IV mental disorders and association with employment status among women in the São Paulo Megacity Mental Health Survey, Brazil $(\mathrm{N}=2,830)$.

\begin{tabular}{|c|c|c|c|c|}
\hline \multirow[t]{4}{*}{ Disorder category/DSM-IV disorder } & \multicolumn{4}{|c|}{ Employment status } \\
\hline & \multirow{3}{*}{$\begin{array}{c}\text { Working * } \\
(n=1,401) \\
\text { Weighted }[\%(S E)]\end{array}$} & \multirow{2}{*}{\multicolumn{3}{|c|}{$\begin{array}{c}\text { Inactive ** } \\
(n=1,119)\end{array}$}} \\
\hline & & & & \\
\hline & & Weighted [\% (SE)] & Crude OR $(95 \% \mathrm{Cl}) * * *$ & Adjusted OR $(95 \% \mathrm{Cl})$ \# \\
\hline Any mental disorder & $34.7(10.80)$ & $30.9(1.80)$ & $0.8(0.68-1.01)$ & $1.1(0.08-1.29)$ \\
\hline Comorbidity (> 1 mental disorders) & $15.5(1.23)$ & $12.3(1.61)$ & $0.8(0.57-1.01)$ & $1.0(0.73-1.32)$ \\
\hline \multicolumn{5}{|l|}{ Mood disorders } \\
\hline Any mood disorder & $15.5(1.23)$ & $12.3(1.60)$ & $0.8(0.56-1.01)$ & $1.0(0.74-1.34)$ \\
\hline Major depression & $14.8(1.30)$ & $10.7(1.55)$ & $0.7(0.50-0.94) \# \#$ & $0.9(0.65-1.22)$ \\
\hline I/II bipolar disorder & $0.5(0.19)$ & $1.4(0.30)$ & $2.6(1.08-6.27) \# \#$ & 3.6 (1.19-11.47) \#\# \\
\hline Dysthymia & $2.1(0.62)$ & $1.9(0.78)$ & $0.9(0.31-2.54)$ & $1.1(0.43-2.37)$ \\
\hline \multicolumn{5}{|l|}{ Anxiety disorders } \\
\hline Any anxiety disorder & $24.8(1.49)$ & $23.0(1.30)$ & $0.9(0.65-1.30)$ & $1.0(0.64-1.52)$ \\
\hline Agoraphobia & $1.8(0.48)$ & $3.1(0.58)$ & $1.7(0.95-3.18)$ & $1.7(0.86-3.26)$ \\
\hline Generalized anxiety disorder & $2.6(0.44)$ & $3.8(0.89)$ & $1.5(0.81-2.73)$ & $1.4(0.83-2.28)$ \\
\hline Obsessive compulsive disorder & $3.5(0.65)$ & $3.6(0.87)$ & $1.0(0.33-3.29)$ & $0.9(0.37-2.01)$ \\
\hline Panic disorder & $1.2(0.26)$ & $1.9(0.60)$ & $1.5(0.79-3.05)$ & $1.7(0.64-4.53)$ \\
\hline Post traumatic stress disorder & $1.4(0.31)$ & $1.8(0.47)$ & $0.9(0.53-1.85)$ & $1.2(0.63-2.13)$ \\
\hline Adult separation anxiety disorder & $2.6(0.55)$ & $2.0(0.50)$ & $0.8(0.38-1.53)$ & $0.9(0.48-1.99)$ \\
\hline Social phobia & $4.6(0.64)$ & $6.0(1.04)$ & $1.3(0.87-2.02)$ & $2.2(1.29-3.63) \# \#$ \\
\hline Specific phobia & $15.5(1.32)$ & $14.0(0.94)$ & $0.9(0.67-1.17)$ & $0.9(0.69-1.30)$ \\
\hline \multicolumn{5}{|l|}{ Impulse-control disordes } \\
\hline Any impulse-control disorder & $3.9(0.65)$ & $3.4(0.73)$ & $0.9(0.51-1.45)$ & $1.3(0.74-2.38)$ \\
\hline Attencion deficit disorder & $0.7(0.23)$ & $0.4(0.18)$ & $0.5(0.16-1.51)$ & $0.5(0.14-2.06)$ \\
\hline Conduct disorder & $0.2(0.15)$ & $0.2(0.15)$ & $1.0(0.15-6.29)$ & - \\
\hline Oppositional defiant disorder & $0.3(0.16)$ & $0.8(0.42)$ & $2.4(0.76-7.79)$ & $6.9(1.47-32.25) \# \#$ \\
\hline Intermittent explosive disorder & $2.9(0.57)$ & $2.6(0.70)$ & $0.9(0.44-1.74)$ & $1.4(0.65-2.99)$ \\
\hline \multicolumn{5}{|l|}{ Substance use disorders } \\
\hline Any substance use disorder & $1.4(0.35)$ & $0.8(0.36)$ & $0.6(0.19-1.70)$ & $0.8(0.24-2.34)$ \\
\hline Alcohol abuse & $6.1(0.33)$ & $0.3(0.24)$ & $0.5(0.05-3.84)$ & $0.6(0.07-5.94)$ \\
\hline Alcohol dependence & $0.6(0.17)$ & $0.2(0.10)$ & $0.3(0.97-1.20)$ & $0.5(0.11-2.17)$ \\
\hline Drug abuse & $0.0(0.01)$ & $0.0(0.01)$ & $2.1(0.11-38.82)$ & $1.1(0.06-18.51)$ \\
\hline Drug dependence & $0.3(0.22)$ & $0.2(0.20)$ & $0.7(0.74-7.11)$ & $1.2(0.06-21.74)$ \\
\hline
\end{tabular}

(continues)

ated with male gender, younger age, higher education and higher income in our study, but there are specific differences when compared to reports from developed countries, where the workforce has higher proportions of middle-aged and more educated individuals 6,8,9,10,11,12. Our findings may reflect the younger age structure and low levels of formal educational attainment in the general Brazilian population.

Overall, for both genders, having 12-month mood disorders is associated with being economically inactive, but it only relates to unemployment among men, and among women, only substance use disorders are associated with being unemployed in our study. As seen in most epidemiological studies, regardless of employment status, higher prevalence rates of mood and anxiety disorders are seen among women, while higher rates of substance use disorders are presented among men 6,8,9,10,11,12, and this gender differences in prevalence rates have not influenced the associations with employment status. In our study, among men, having any mental disorders, any mood disorders, I/II bipolar disor- 
Table 3 (continued)

\begin{tabular}{|c|c|c|c|}
\hline \multirow[t]{2}{*}{ Disorder category/DSM-IV disorder } & \multicolumn{3}{|c|}{$\begin{array}{l}\text { Employment status } \\
\text { Unemployed }(n=310)\end{array}$} \\
\hline & Weighted [\% (SE)] & Crude OR $(95 \% \mathrm{Cl}) * * *$ & Adjusted OR $(95 \% \mathrm{Cl})$ \# \\
\hline Any mental disorder & $33.0(0.84)$ & $0.8(0.64-1.13)$ & $0.8(0.60-1.17)$ \\
\hline Comorbidity (> 1 mental disorders) & $13.2(2.28)$ & $0.8(0.51-1.34)$ & $0.8(0.51-1.29)$ \\
\hline \multicolumn{4}{|l|}{ Mood Disorders } \\
\hline Any mood disorder & $12.3(1.78)$ & $0.8(0.51-1.26)$ & $0.8(0.51-1.23)$ \\
\hline Major depression & $12.3(1.78)$ & $0.8(0.52-1.23)$ & $0.77(0.51-1.18)$ \\
\hline I/II bipolar disorder & $0.1(0.14)$ & $0.3(0.02-3.36)$ & $0.3(0.02-5.13)$ \\
\hline Dysthymia & $1.3(0.6)$ & $0.6(0.16-2.11)$ & $0.6(0.18-2.34)$ \\
\hline \multicolumn{4}{|l|}{ Anxiety disorders } \\
\hline Any anxiety disorder & $20.9(3.13)$ & $0.9(0.48-1.76)$ & $0.9(0.46-1.76)$ \\
\hline Agoraphobia & $2.3(0.86)$ & $1.3(0.60-2.82)$ & $1.2(0.56-2.60)$ \\
\hline Generalized anxiety disorder & $2.4(0.81)$ & $0.9(0.36-2.32)$ & $1.1(0.43-2.76)$ \\
\hline Obsessive compulsive disorder & $3.0(1.23)$ & $0.9(0.29-2.83)$ & $0.8(0.23-2.53)$ \\
\hline Panic disorder & $2.4(1.33)$ & $2.0(0.55-7.55)$ & $2.1(0.73-6.24)$ \\
\hline Post traumatic stress disorder & $2.7(0.98)$ & $1.8(0.78-4.01)$ & $1.9(0.84-4.54)$ \\
\hline Adult separation anxiety disorder & $1.7(0.65)$ & $0.6(0.22-1.75)$ & $0.6(0.20-1.87)$ \\
\hline Social phobia & $3.3(1.46)$ & $0.7(0.29-1.75)$ & $0.7(0.32-1.48)$ \\
\hline Specific phobia & $12.5(2.84)$ & $0.8(0.67-1.17)$ & $0.81(0.41-1.32)$ \\
\hline \multicolumn{4}{|l|}{ Impulse-control disordes } \\
\hline Any impulse-control disorder & $6.2(2.48)$ & $1.6(0.64-4.14)$ & $1.4(0.56-3.65)$ \\
\hline Attencion deficit disorder & $0.6(0.36)$ & $0.8(0.22-2.58)$ & $0.6(0.16-2.19)$ \\
\hline Conduct disorder & $0.2(0.001)$ & - & - \\
\hline Oppositional defiant disorder & $0.0(0.01)$ & $0.3(0.03-2.70)$ & $0.3(0.02-3.75)$ \\
\hline Intermittent explosive disorder & $5.5(2.27)$ & $1.9(0.65-5.72)$ & $1.7(0.62-4.88)$ \\
\hline \multicolumn{4}{|l|}{ Substance use disorders } \\
\hline Any substance use disorder & $3.2(1.15)$ & $2.3(1.02-5.09) \# \#$ & $2.1(0.73-6.09)$ \\
\hline Alcohol abuse & $1.6(0.82)$ & $2.6(0.57-11.68)$ & $2.2(0.28-17.26)$ \\
\hline Alcohol dependence & $0.7(0.44)$ & $1.2(0.28-5.13)$ & $1.1(0.27-4.70)$ \\
\hline Drug abuse & $0.5(0.46)$ & $12.5(0.83-185.90)$ & $3.8(0.40-36.08)$ \\
\hline Drug dependence & $0.6(0.13)$ & $1.9(0.26-13.16)$ & $3.7(0.37-36.19)$ \\
\hline
\end{tabular}

95\%Cl: 95\% confidence interval; DSM-IV: Diagnostic and Statistical Manual of Mental Disorders, $4^{\text {th }}$ edition; OR: odds ratio; SE: standard error.

* Employed, self-employed, owner; OR = 1 (base-line);

** Homeworkers, students, disabled, retirees, sickness benefits;

$* * *$ OR cruded comparing inactive vs working;

\# OR adjusted by sociodemographics characteristics comparing inactive vs working; OR obtained by multinomial logistic regression using the category

"working" as the reference;

\#\# p-value $<0.05$.

der, and any anxiety disorders (especially panic and post-traumatic stress) was associated with being economically inactive; and the occurrence of any 12-month mood disorders (major depression and bipolar I/II disorder) increased the chances of being unemployed. Among women, having 12-month bipolar disorder, social phobia and oppositional defiant disorder was associated with being economically inactive, and the diagnosis of any substance use disorder in the past 12-months was associated with being unemployed (crude analyses). Regarding comorbidity, having more than one mental disorder was associated with being economically inactive and unemployed among men, but was not associated to current employment status in women. We have decided to analyze the associations of mental disorders with employment status separately by gender, as there are different characteristics of work attainment for men and women in Brazil, reflecting our social and cultural backgrounds related to gender traditional roles. The economically inactive group was mainly composed of women 


\section{Table 4}

Use of health services by employment status of respondents with 12-month DSM-IV mental disorders in the São Paulo Megacity Mental Health Survey, Brazil $(\mathrm{N}=1,395)$.

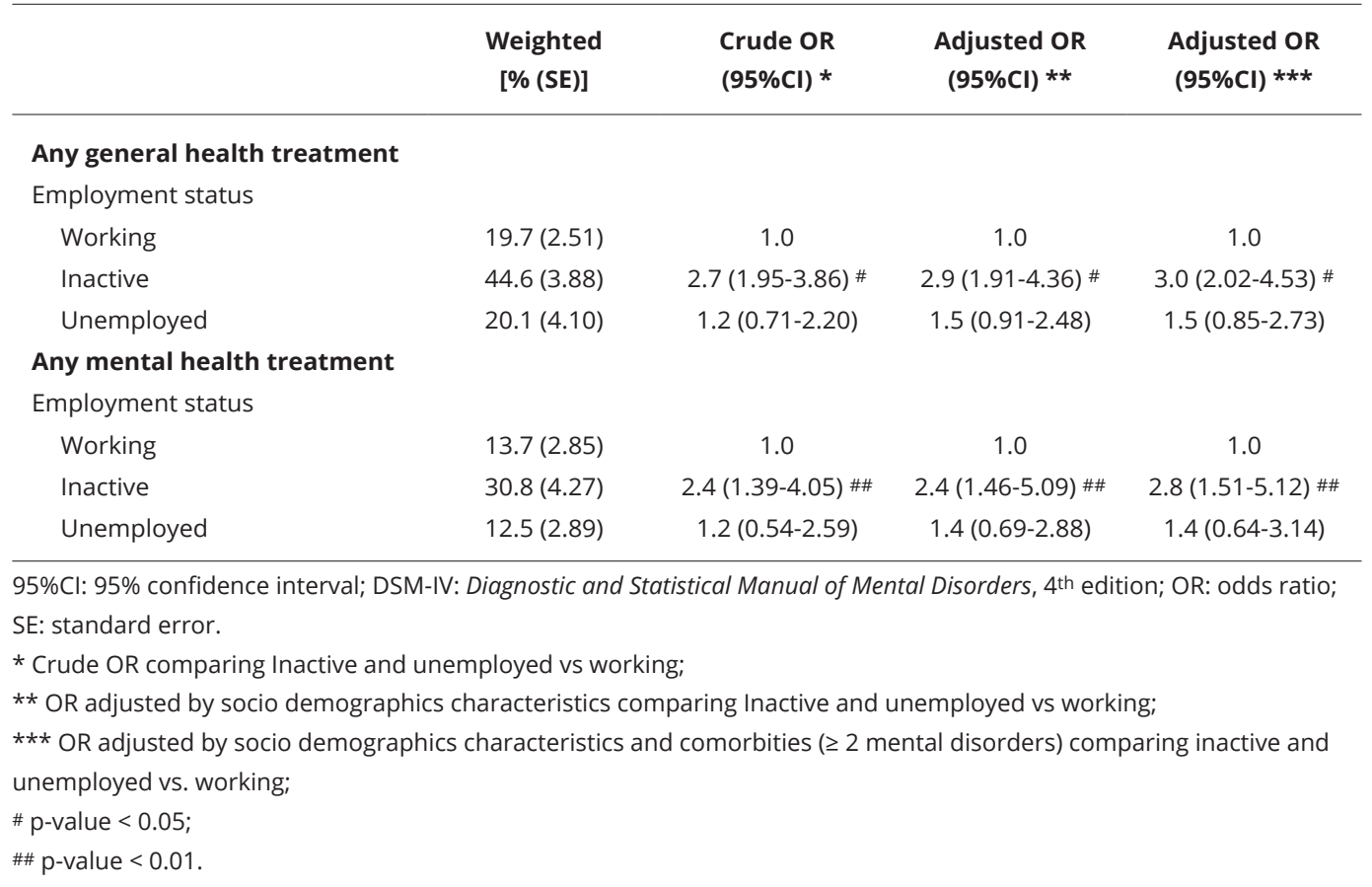

( $\mathrm{n}=1,119 ; 75.3 \%$ ), and $99.7 \%$ of those self-classified as homeworkers were women (data not shown herein). This assumption was corroborated when we formally tested the interaction between gender and the associations of mental disorders with employment status. This analytical strategy, however, makes it difficult to compare our results with those from other reports, as most studies analyzed men and women altogether, in the same group 7,8,9,10,11,12,13,14,15,16,17.

Chong et al. 7 assessed the association between $12 \mathrm{~m}-\mathrm{MD}$ and work disability in Singapore $(\mathrm{n}=$ 6,429 ), where $71 \%$ were working, $24.5 \%$ economically inactive and $4.5 \%$ unemployed, similar to our study, but smaller rates of any $12 \mathrm{~m}-\mathrm{MD}$ were found ( $2.3 \%$ among the working, $1 \%$ among the economically inactive, and 5.3\% among the unemployed); although they have not evaluated associations between mental disorders and employment status, they reported high rates of work-related disability among employees with mental disorders 7 .

It is well established that there are higher prevalence rates of mental disorders among the unemployed population compared to those who are in the workforce 29.30 , and this association is likely to be two-way, with mental problems leading to unemployment and, also, occurring as detrimental social and/or psychological consequences of unemployment 9,18,19,30. Indeed, individuals with mental disorders are less likely to enter workforce and to remain employed, and may have greater difficulty to reenter the workforce, whether due to disability, rehabilitation or any other negative consequences 30 . Even those who start working often have lower income wages, and present qualitative and quantitative reduction in work performance 6,7,8,9,10,11,12,13,14,15,16. The relationship between mental disorders, work losses, work inactivity and unemployment has, therefore, a complex nature 6,7,10,11,12,13,14,15,16.

The magnitude of work losses related to the growing number of individuals unemployed and unable to work due to mental disorders has been used to estimate the burden of mental disorders on workers and their families, employers and on the whole economy. This involves not only individual financial losses, but also individual suffering and family emotional and financial burden, as well as societal load, since absenteeism and presenteeism heavily impact employers and social welfare systems $6,8,9,11,16,21,29,30,31,32$. 
Regarding use of health services, the early detection and adequate treatment are important steps to reduce the impact and burden of mental disorders in general, and specifically related to employment status, they may be strong determinants of returning to the workforce 29,30 . In our study, treatment seeking among those suffering from $12 \mathrm{~m}-\mathrm{MD}$ was more frequent among the economically inactive, both in general and mental health services, adjusting for sociodemographic characteristcs and psychiatric comorbidities. This may reflect the composition of this sub-sample, as respondents on sick leave and early retired due to disability were categorized as economically inactive in our study.

On the other hand, although those unemployed presented higher rates of specific mental disorders, treatment seeking was not more frequent among them compared to those working, and this may indicate a poorer access to general and specialized services among the unemployed population $33,34,35,36$, which may retard or even prevent return to the workforce. These findings are generally reproduced in other studies. Treatment seeking was only reported by $19.2 \%$ of workers and $7.6 \%$ of economically inactive individuals with major depression in Singapore, and those with alcohol abuse and dependence, independently of employment, did not seek treatment at all 7 . In a previous report from the WMH surveys, most respondents with mental disorders have not received any kind of treatment in the previous 12-month period, although a positive relationship between mental disorder severity and treatment seeking was observed 24,37,38. Nevertheless, healthcare treatment seeking among the São Paulo Metropolitan Area population was much less frequent than reported in highincome countries 39 .

Our study has not specifically evaluated the use of healthcare services related to severity of mental disorders and its relationship with employment status, but among respondents with mental disorders, those unemployed do not usually seek healthcare services. This may indicate a treatment gap due to the lack of health insurance or insufficient resources to fund private care, as well as a poor coverage by the public health sector. Specifically related to employment, the lower demand for treatment among working respondents may also reflect the influence of the working atmosphere, which discourages medical leave and recognition of mental health symptoms, and reinforces refusal to accept a psychiatric diagnosis, due to fear of stigma, job loss and discrimination 36. Previous studies showed that low perceived need and attitudinal barriers are the major barriers to treatment seeking and staying in treatment among individuals with common mental disorders worldwide, regardless of their employment status 40. Apart from targeting structural barriers, mainly in countries with poor resources, improving mental health literacy in the population and addressing attitudinal barriers and stigma are important endeavors 31,36,40.

Finally, the interpretation of findings reported in this paper should take into account a number of limitations, mostly related to the cross-sectional nature of the survey. The associations of mental disorders with employment status observed should be interpreted in a bidirectional manner, limiting inferences of causality. Although we used a standardized psychiatric assessment tool, DSM-IV diagnoses were based on self-reported retrospective information concerning symptoms occurred over the past 12-month period, with no clinical gold-standard assessment to check the consistency of diagnostic procedures. However, the comparison between self-report symptoms and medical records has shown moderate to reasonable agreement in other studies 41,42,43. The concordance of the CIDI 3.0 with the Structured Clinical Interview for DSM-IV (SCID) was also unbiased for 12-month prevalence estimates, and the reliability of a previous Brazilian version of the CIDI (CIDI 2.1) showed high kappa values, ranging from 0.76 (for OCD) to 1.0 (for alcohol and substance-related disorders) 44.

Selection bias may have contributed to underestimate the actual prevalence rates, as individuals suffering from more severe mental disorders are less likely to participate in household surveys of this sort and hospitalized patients, homeless people and individuals living in institutions are not eligible to participate. Furthermore, respondents with more severe mental or physical conditions living at home were more likely to be excluded from selection as ineligible to participate in such a complex assessment (due to impeding physical, mental or cognitive conditions) or they may present differential mortality 26,45. Although the CIDI 3.0 was rebuilt considering several strategies to minimize recall bias and information bias while approaching sensitive issues or preceding information 27 , it is unlikely that they were completely ruled out. While employment status was assessed at the time of the interview, mental disorders and treatment seeking were assessed over the previous 12 -month period. Moreover, the overall use of any general and mental health care services over the 
past 12 months was assessed, and it may or may not have been related to the specific mental health problems identified.

\section{Conclusion}

Understanding the complex relationship between employment status and mental disorders and the influence of employment status upon the use of health care services are essential steps to create improved local public health and welfare policies, reinforcing the need for adequate destination of usually scarce resources. Initiatives to promote inclusion in the workforce should be considered a priority in Brazil, together with individual interventions aiming at preventing the burden of mental disorders, such as disability, sickness leaves or benefits, absenteeism and presenteeism, from longterm sickness absence to permanent disability, causing high financial costs to individuals, to their families, to their workplaces, and to the social security system in Brazil.

These approaches are necessary and their advocacy can be grounded on findings from populationbased studies such as this, reported herein. New types of interventions in different contexts should be considered and tested. This study demonstrates that poor mental health is associated with being economically inactive or unemployed, with significant gender differences. These findings highlight the cyclical and potentially entrenched nature and consequences of poor mental health and emphasize the need for a tuned approach involving employment, welfare and mental health policies.

\section{Contributors}

M. H. França conceived and designed the experiments, performed the experiments, analyzed the data, and wrote the paper. S. M. Barreto performed the experiments, analyzed the data, and wrote the paper. F. G. Pereira conceived and designed the experiments, she also analyzed the data and wrote the paper. L. H. S. G. Andrade conceived and designed the experiments, and wrote the paper. M. C. A. Paiva wrote and revised the paper. M. C. Viana conceived and designed the experiments, performed the experiments, analyzed the data, and wrote the paper.

\section{Acknowledgments}

The São Paulo Megacity Mental Health Survey was carried out with the World Health Organization World Mental Health (WMH) Survey Initiative. We thank the Graduate Program in Public Health at Federal University of Espírito Santo (UFES) staff members, M. C. Viana, F. G. Pereira and M. C. A. Paiva. Thanks also to the WMH staff for database. A complete list of WMH publications can be found at http:// www.hcp.med.harvard.edu/wmh/. To FAPESP and FACITEC for the financial support.

\section{References}

1. Eaton WW, Martins SS, Nestadt G, Bienvenu OJ, Clarke D, Alexandre P. The burden of mental disorders. Epidemiol Rev 2008; 30:1-14.

2. Patel V, Chisholm D, Parikh R, Charlson FJ, Degenhardt L, Dua T, et al. Addressing the burden of mental, neurological, and substance use disorders: key messages from Disease Control Priorities, 3rd edition. Lancet 2016; 387:1672-85.

3. Schramm JMA, Oliveira AF, Meite IC, Valente JG, Gadelha AMJ, Portela MC, et al. Transição epidemiológica e o estudo de carga de doença no Brasil. Ciênc Saúde Coletiva 2004; 9:897908.

4. Schmidt MI, Duncan BB, Azevedo e Silva G, Menezes AM, Monteiro CA, Barreto SM, et al. Chronic non-communicable diseases in Brazil: burden and current challenges. Lancet 2011; 377:1949-61.

5. Alonso J, Petukhova M, Vilagut G, Chatterji S, Heeringa S, Üstün TB, et al. Days out of role due to common physical and mental conditions: results from the WHO World Mental Health Surveys. Mol Psychiatry 2011; 16:1234-46.

6. Graaf R, Tuithof M, van Dorsselaer S, Ten Have M. Comparing the effects on work performance of mental and physical disorders. Soc Psychiatry Psychiatr Epidemiol 2012; 47:1873-83. 
7. Chong SA, Vaingankar JA, Abdin E, Subramaniam M. Mental disorders: employment and work productivity in Singapore. Soc Psychiatry Psychiatr Epidemiol 2013; 48:117-23.

8. Lim D, Sanderson K, Andrews G. Lost productivity among full-time workers with mental disorders. J Ment Health Policy Econ 2000; 3:139-46.

9. Ford E, Clark C, McManus S, Harris J, Jenkins $\mathrm{R}$, Bebbington $\mathrm{P}$, et al. Common mental disorders, unemployment and welfare benefits in England. Public Health 2010; 12412:675-81.

10. Kessler RC, Akiskal HS, Ames M, Birnbaum H, Greenberg P, Hirschfeld RM, et al. Prevalence and effects of mood disorders on work performance in a nationally representative sample of US workers. Am J Psychiatry 2006; 163: 1561-8.

11. Kessler R, Frank G. The impact of psychiatric disorders on work loss days. Psychol Med 1997; 27:861-73.

12. Sanderson K, Andrews G. Common mental disorders in the workforce: recent findings from descriptive and social epidemiology. Can J Psychiatry 2006; 51:63-75.

13. Chwastiak LA, Von Korff M. Disability in depression and back pain: evaluation of the World Health Organization Disability Assessment Schedule (WHODAS II) in a primary care setting. J Clin Epidemiol 2003; 56:507-14.

14. Kessler RC, Greenberg PE, Mickelson KD, Meneades LM, Wang PS. The effects of chronic medical conditions on work loss and work cutback. J Occup Environ Med 2011; 43: 218-25.

15. Bijl RV, Ravelli A. Current and residual functional disability associated with psychopathology. Psychol Med 2000; 30:657-68.

16. Ervasti J, Vahtera J, Pentti J, Oksanen T, Ahola K, Kivimäki M, et al. Depression-related work disability: socioeconomic inequalities in onset, duration and recurrence. PLoS One 2013; 8:e79855.

17. Broadhead WE, Blazer DG, George LK, Kit Tse C. Depression, disability days, and days lost from work in a prospective epidemiologic survey. JAMA 1990; 264:2524-8.

18. Andrade LH, Baptista MC, Alonso J, Petukhova M, Bruffaerts R, Kessler RC, et al. Days outof-role due to common physical and mental health problems: Results from the São Paulo megacity mental health survey. Clinics 2013; 68:1392-9.

19. Lahelma E. Unemployment and mental wellbeing: elaboration of the relationship. Int J Health Serv 1992; 22:261-74.

20. Scheid T. An investigation of work and unemployment among psychiatric clients. Int J Health Serv 1993; 23:763-82.

21. Zhang M, Rost K, Fortney J, Smith R. A community study of depression treatment and employment. Psychiatr Serv 1999; 50:1209-13.

22. Silva JS, Fischer FM. Adoecimento mental incapacitante: benefícios previdenciários no Brasil entre 2008-2011. Rev Saúde Pública 2014; 48:186-90.
23. Andrade LH, Viana MC, Tofoli LF, Wang YP. Influence of psychiatric morbidity and sociodemographic determinants on use of service in a catchment area in the city of São Paulo, Brazil. Soc Psychiatry Psychiatr Epidemiol 2008; 43:45-53.

24. Andrade LH, Wang Y-P, Andreoni S, Silveira CM, Alexandrino-Silva C, Siu ER, et al. Mental disorders in megacities: findings from the São Paulo Megacity Mental Health Survey, Brazil. PLoS One 2012; 2012;7:e31879.

25. Viana MC, Andrade LH. Lifetime prevalence, age and gender distribution and age-of-onset of psychiatric disorders in the São Paulo Metropolitan Area, Brazil: results from the São Paulo Megacity Mental Health Survey. Rev Bras Psiquiatr 2012; 34:249-60.

26. Viana MC, Teixeira M, Beraldi F, Bassani I, Andrade LH. São Paulo Megacity Mental Health Survey: a population-based epidemiological study of psychiatric morbidity in the São Paulo Metropolitan Area. Aims, design and field implementation. Rev Bras Psiquiatr 2009; 31:375-86.

27. Kessler RC, Ustun TB. The World Mental Health (WMH) survey initiative version of the World Health Organization (WHO) Composite International Diagnostic Interview (CIDI). Int J Methods Psychiatr Res 2004; 13:93-121.

28. Ministério do Trabalho e Emprego. Evolução do salário mínimo. http://trabalho.gov.br/im ages/Documentos/SalarioMinimo/EVOLEIS M1940a2016.pdf (accessed on 30/Nov/2016).

29. Olesen SC, Butterworth P, Leach LS, Kelaher M, Pirkis J. Mental health affects future employment as job loss affects mental health: findings from a longitudinal population study. BMC Psychiatry 2013; 113:144.

30. Claussen B, Bjorndal A, Hjort P. Health and reemployment in a two year follow up of long term unemployed. J Epidemiol Community Health 1993; 47:14-8.

31. North FM, Syme SL, Feeney A, Shipley M, Marmot M. Psychosocial work environment and sickness absence among British civil servants: the Whitehall II study. Am J Public Health 1996; 86:332-40.

32. Van Dongen CJ. Quality of life and self-esteem in working and non working persons with mental illness. Community Ment Health J 1996; 32:535-48.

33. Pessoto UC, Heimann LS, Boaretto RC, Castro IE, Kayano J, Ibanhes LC, et al. Health care services utilization and access inequalities in the Sao Paulo Metropolitan Region. Ciênc Saúde Coletiva 2007; 12:351-62.

34. Nascimento AF, Galvanese AT. Evaluation of psychosocial healthcare services in the city of São Paulo, South eastern Brazil. Rev Saúde Pública 2009; 43 Suppl 1:8-15.

35. Hämäläinen J, Isometsä E, Laukkala T, Kaprio J, Poikolainen K, Heikkinen M, et al. Use of health services for major depressive episode in Finland. J Affect Disorders 2004; 79:105-11. 
36. Burns T, Eichenberger A, Eich D, AjdacicGross V, Angst J, Rössler W. Which individuals with affective symptoms seek help? Results from the Zurich epidemiological study. Acta Psychiatr Scand 2003; 108:419-26.

37. Wang PS, Aguilar-Gaxiola S, AlHamzawi AO, Alonso J, Andrade LH et al. Treated and untreated prevalence of mental disorder worldwide. In: Thornicroft G, Szmukler G, Mueser $\mathrm{K}$, Drake B, editors. Oxford textbook of community mental health. Oxford: Oxford University Press; 2011. p. 50-66.

38. Demyttenaere K, Bruffaerts R, Posada-Villa J, Gasquet I, Kovess V, Lepine JP, et al. Prevalence, severity, and unmet need for treatment of mental disorders in the World Health Organization World Mental Health Surveys. JAMA 2004; 291:2581-9.

39. Wang YP, Chiavegatto Filho AD, Campanha AM, Malik AM, Mogadouro MA, Cambraia M, et al. Patterns and predictors of health service use among people with mental disorders in São Paulo metropolitan area, Brazil. Epidemiol Psychiatr Sci 2016; 12:1-13.

40. Andrade LH, Alonso J, Mneimneh Z, Wells JE, Al-Hamzawi AO, Borges G, et al. Barriers to mental health treatment: results from the WHO World Mental Health (WMH) Surveys. Psychol Med 2013; 9:1-15.
41. National Center for Health Statistics. Evaluation of national health interview survey diagnostic reporting. Hyattsville: Department of Health and Human Services; 1994. (Series 2: Data Evaluation and Methods Research).

42. Baker MM, Stabile M, Deri C. What do self-reported, objective measures of health measure? Cambridge: National Bureau of Economic Research; 2001.

43. Knight M, Stewart-Brown S, Fletcher L. Estimating health needs: the impact of a checklist of conditions and quality of life measurement on health information derived from community surveys. J Public Health Med 2001; 23: 179-86.

44. Quintana MI, Andreoli SB, Jorge MR, Gastal FL, Miranda CT. The reliability of the Brazilian version of the Composite International $\mathrm{Di}$ agnostic Interview (CIDI 2.1). Braz J Med Biol Res 2004; 37:1739-45.

45. Eaton WW, Anthony JC, Tepper S, Dryman A. Psychopathology and attrition in the Epidemiologic Catchment Area Study. Am J Epidemiol 1992; 135:1051-9. 


\section{Resumo}

Os transtornos mentais estão associados à situação laboral enquanto preditores significativos $e$ consequências do desemprego e da aposentadoria precoce. Este estudo estima as taxas de prevalência de transtornos mentais nos últimos 12 meses de acordo com o DSM-IV, além do uso de serviços de saúde e as associações com situação laboral de acordo com gênero na Região Metropolitana de São Paulo, Brasil. Foram analisados dados do São Paulo Megacity Mental Health Survey $(n=$ 5.037), um estudo populacional que avalia a prevalência e determinantes dos transtornos mentais entre adultos, usando a Composite International Diagnostic Interview. As associações foram estimadas através de razões de prevalência, obtidas por regressão logística binomial e multinomial. $O$ estudo mostra que os transtornos mentais, especialmente transtornos de humor estão associados à inatividade ou ao desemprego entre os homens $e$ à inatividade entre as mulheres, com exceção aos transtornos devido ao uso de substâncias psicoativas que também estão associados ao desemprego em as mulheres. Entre os portadores de transtornos mentais, a busca de serviços de saúde é menos frequente entre os desempregados.

Transtornos Mentais; Emprego; Serviços de Saúde

\section{Resumen}

Los trastornos mentales están asociados a la situación laboral, como predictores significativos por las consecuencias que provocan el desempleo y la jubilación precoz. Este estudio estima las tasas de prevalencia de trastornos mentales en 12 meses, de acuerdo con el DSM-IV, además del uso de servicios de salud y las asociaciones existentes con la situación laboral, de acuerdo con el género en el área metropolitana de São Paulo, Brasil. Se analizaron los datos del São Paulo Megacity Mental Health Survey $(n=5.037)$, un estudio poblacional que evalúa la prevalencia y determinantes de los trastornos mentales entre adultos, usando la Composite International Diagnostic Interview. Las asociaciones se estimaron con razones de prevalencia, obtenidas por la regresión logística binomial y multinomial. El estudio muestra que los trastornos mentales, especialmente los trastornos de humor, están asociados a la inactividad $o$ al desempleo en hombres y a la inactividad en mujeres, pero que sólo el trastorno de consumo de sustancias está asociado al desempleo en mujeres. Entre los portadores de trastornos mentales, la búsqueda de servicios de salud es menos frecuente entre los desempleados.

Trastornos Mentales; Empleo; Servicios de Salud
Submitted on $05 /$ Sep/2016

Final version resubmitted on 08/Nov/2016

Approved on 28/Nov/2016 